\title{
The effect of coexistence between larvae of Anopheles gambiae and Culex quinquefasciatus on larvicidal efficacy of Bacillus thuringiensis var. israelensis
}

\author{
Yahya A. Derua ${ }^{a, b^{*}}$, Eliningaya J. Kweka ${ }^{c, d}$, William N. Kisinzae, Guiyun Yan, Andrew K. Githekof, \\ Franklin W. Mosha ${ }^{a}$
}

Kilimanjaro Christian Medical University College, Tumaini University Makumira, P. O. Box 2240, Moshi, Tanzania

'vational Institute for Medical Research, Amani Research Centre, P. O. Box 81, Muheza, Tanga, Tanzania

¿Division of Livestock and Human Diseases Vector Control, Tropical Pesticides Research Institute, P.O. Box 3024, Arusha, Tanzania

Department of Medical Parasitology and Entomology, Catholic University of Health and Allied Sciences, P.O. Box 1464, Mwanza, Tanzania

eProgram in Public Health, College of Health Sciences, University of California, Irvine, CA 92697, USA

${ }^{\dagger}$ Climate and Human Health Research Unit, Centre for Global Health Research, Kenya Medical Research Institute, P. O. Box 1578, Kisumu, Kenya

"Corresponding author to Yahya Athumani Derua, P. O. Box 81, Muheza, Tanga, Tanzania, Phone numbers: +255784508697, E-mail address: yaderua@gmail.com

\begin{abstract}
Background: The efficacy of Bacillus thuringiensis var. israelensis (Bti) is affected by several factors including the species of the mosquito. Mosquito larvae of different species are found to coexist in larval breeding habitats. This study evaluated whether the coexistence between Anopheles gambiae and Culex quinquefasciatus affect the larvicidal activity of Bti.
\end{abstract}

Methods: Two parallel larval bioassay experiments were conducted to test A. gambiae sensu stricto (s.s) and C. quinquefasciatus larvae susceptibility to Bti. They were followed by three parallel bioassays in which A. gambiae s.s and C. quinquefasciatus larvae were mixed in different proportions such that the earlier species contributed three quarters, half and a quarter of the larvae in each testing cup respectively. In each bioassay, six Bti concentrations were tested in four replicates and repeated on three different days. Larvae mortality was scored 24 hours after application of Bti and subjected to Probit analysis.

Results: $C$. quinquefasciatus was significantly more susceptible to Bti than $A$. gambiae s.s at both lethal concentration values ( $\left(\mathrm{C}_{50}\right.$ and $L \mathrm{C}_{95}$ ). In coexisting scenario, $\mathrm{LC}_{50}$ of Bti was significantly lower when the proportion of $C$. quinquefasciatus exceeded $50 \%$. No significant variation in susceptibility to Bti was observed at $L_{9}$ in any proportion of coexistence between the two species.

Conclusion: The findings show that larvae of $C$. quinquefasciatus were significantly more susceptible to Bti than those of $A$. gambiae s.s. Moreover, when larvae of the two species coexisted, there was a general trend of increase in sensitivity to Bti with higher proportion of $C$. quinquefasciatus. Although this increase in sensitivity of coexisting larvae to $B t i$ is worth noting, our findings suggest that it will not impact larval control where A. gambiae s.s and C. quinquefasciatus coexist.

Keywords: Anopheles gambiae sensu stricto, Culex quinquefasciatus, Bacillus thuringiensis var. israelensis, Larval bioassays. 


\section{INTRODUCTION}

Tnsecticide-based malaria vector control Iinterventions have contributed significantly to the reduction of global malaria transmission and consecutively renewed interest in global malaria elimination. ${ }^{1}$ However, new novel tools are urgently needed not only to complement the core malaria vector control interventions (insecticide treated nets and indoor residual spraying) but also with the potential to tackle threats posed by insecticide resistance and behavioral adoptions by malaria vectors. $^{2}$ Application of bacterial larvicide products based on Bacillus thuringiensis var. israelensis (Bti) and Bacillus sphaericus (Bs) has been found to be effective and with potential to control both indoor and outdoor biting malaria vectors and possibly delay the evolution of insecticide resistance. ${ }^{2}$ Furthermore, the potential role of larviciding for malaria vector control increases as malaria continues in a declining trend, creating a more appropriate condition for the interventions as summarized elsewhere. ${ }^{3}$

Bacterial larvicide Bacillus thuringiensis var. israelensis (Bti) (Bacillales: Bacillaceae) is grampositive, spore-forming aerobic bacteria isolated from a multitude of aquatic larval habitats. ${ }^{4}$ It has been used extensively as a larvicide for mosquito (Diptera: Culicidae) and black fly (Diptera: Simuliidae) control globally. ${ }^{4,5}$ The larvicidal activity of Bti is based on delta-endotoxins produced by this bacterium at the time of sporulation. ${ }^{5}$ When ingested by susceptible mosquito larvae, these toxins bind to the surface membranes of the epithelial cells of the larval midgut disrupting osmotic balance and resulting in the death of the larvae. ${ }^{5}$ Based on review of bacterial larvicides, $13 \mathrm{Bti}$ based products have been evaluated and found to be effective for malaria vector control in subSaharan Africa (SSA). ${ }^{3}$ However, only the Bti strain AM65-52 (Vectobac ${ }^{\circledR}$ granules (GR) and VectoBac ${ }^{\circledR}$ water dispersible granules (WG)) has been prequalified by the World Health Organization (WHO) to be used for malaria vector control. ${ }^{6}$ In addition to its proven effectiveness in mosquito control, Bti is generally safe to other non-target organisms coexisting with mosquito larvae in aquatic habitats. ${ }^{7}$ The reported efficacy and safety of Bti when used for malaria vector control make it ideal for inclusion in Integrated Vector Management (IVM) operations to supplement other vector control strategies. ${ }^{3}$ A study conducted in western Kenya demonstrated the potential role of integrating larviciding into adult malaria mosquito vector control interventions in reducing malaria transmission. ${ }^{8}$
Efficacy of Bti in mosquito control has been reported to vary greatly, mainly due to factors related to target mosquitoes (species of mosquito, their respective feeding strategies, age and density of larvae), larval habitat conditions (temperature, solar radiation, depth of water, turbidity, organic contents and presence of vegetation) and larvicide properties (application rates, toxin contents, type of carrier, how effective the material reach the target, settling rate, means of application and frequency of treatment). ${ }^{4,5}$ In this regard, control efficacy and persistence of Bti based products have been shown to vary greatly in different ecological settings in SSA. ${ }^{3}$ Understanding factors that cause variation of the effectiveness of Bti is important, particularly when planning larviciding interventions in different geographical settings. Like any other larviciding intervention, the Bti application must be guided by adequate knowledge of the prevailing mosquito vectors species, including their ecology, and the properties of the larvicide used. ${ }^{5}$

Studies have reported contradictory results on the level of susceptibility of larvae of Anopheles gambiae complex (Diptera: Culicidae) and Culex species (Diptera: Culicidae) to Bti. In controlled conditions, studies have shown that these two-mosquito species are equally susceptible to $B t i .^{9-11}$ On the other hand, in laboratory settings, Culex quinquefasciatus (Diptera: Culicidae) larvae were reported to be up to 4 times more susceptible to Bti than A. gambiae complex. ${ }^{12} \mathrm{~A}$ field study conducted on the Kenyan coast showed that $A$. gambiae complex were more susceptible to $B t i$

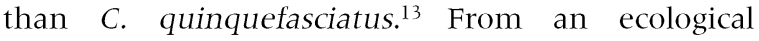
perspectives, it has been documented that the larvae of A. gambiae complex spend much more time feeding on the water surface whereas $C$. quinquefasciatus larvae feed under the surface of the water. ${ }^{14}$ The nature and properties of a particular larvicide (including its settling rate) is likely to affect the larvicidal exposure rate between surface and bottom feeding larvae. ${ }^{15}$

Immature stages (larvae and pupae) of $A$. gambiae complex and $C$. quinquefasciatus have been found to coexist freely in the natural larval habitats. ${ }^{16,17}$ In some instances, the coexistence of A. gambiae complex and C. quinquefasciatus has been detected in all potential anopheline larval habitats surveyed. ${ }^{16}$ This coexistence has shown to harm the fitness of resulting adult mosquitoes due to competition for resources. ${ }^{18}$ The coexistence may lead to competition for available larvicide toxins in a treated larval habitat which may subsequently impact on the effectiveness of Bti. It has been shown that $C$. quinquefasciatus has a relatively 
higher particulate filtration rate than $A$. gambiae. ${ }^{15}$ In the coexistence scenario, it is assumed that $C$. quinquefasciatus may filter more Bti toxins due to its inherent effective feeding behaviour and will succumb more to the lethal effect of the larvicide than $A$. gambiae complex. The current study was designed to evaluate whether co-existence between $A$. gambiae sensu stricto (s.s) and C. quinquefasciatus in the same larval habitat could influence the larvicidal activity of Bti.

\section{MATERIALS AND METHODS}

\section{Study area and test mosquitoes}

The study was conducted at the insecticide testing facility of the National Institute for Medical Research, Amani Medical Research Centre in Muheza, Tanga, Tanzania. Larvae of A. gambiae s.s (Kisumu strain) and $C$. quinquefasciatus (Tropical Pesticide Research Institute stain), both of which were fully susceptible to insecticides were used for the laboratory bioassays. The tested colony of A. gambiae s.s has been maintained for 30 generations at the insectary of the Amani Medical Research Centre whereas $C$. quinquefasciatus (at their $40^{\text {th }}$ generation) were obtained from the Tropical Pesticide Research Institute, Arusha, Tanzania. A. gambiae s.s (Kisumu strain) is a reference strain, considered susceptible to insecticides and has been used extensively in bioassay experiments across Africa. ${ }^{19}$

Adult A. gambiae s.s and $C$. quinquefasciatus were maintained at an average room temperature of $26.5^{\circ} \mathrm{C}$ $\left(24-29^{\circ} \mathrm{C}\right)$ and an average relative humidity of $77.5 \%$ $(65-90 \%)$ whereas larvae were kept at an average room temperature of $32{ }^{\circ} \mathrm{C}\left(29-35^{\circ} \mathrm{C}\right)$. Before larval bioassays, first and second stage (early instar) larvae of A. gambiae s.s were fed on instant yeast (Istanbul, Turkey) whereas third and fourth stage (late instars) were fed Aquafin ${ }^{\circledR}$ fish food (Quanzhou, China). Larvae of $C$. quinquefasciatus were fed Whiskas ${ }^{\circledR}$ cat food (Mars Africa, South Africa). The feeding strategy was such that, $0.25-0.5 \mathrm{~g}$ of instant yeast was reconstituted in $4 \mathrm{~mL}$ of water and then $1 \mathrm{~mL}$ solution was added to $1500 \mathrm{~mL}$ chlorine free tap water, which was sufficient to feed around 500 respective first and second stage larvae of A. gambiae s.s for one day. For third and fourth stage larvae of this species, $0.1 \mathrm{~g}$ Aquafin $^{\circledR}$ was added to $1500 \mathrm{~mL}$ chlorine free tap water to feed around 500 larvae per day. For $C$. quinquefasciatus, $1 \mathrm{~g}$ of Whiskas ${ }^{\circledast}$ cat food was added in $1500 \mathrm{~mL}$ of chlorine free tap water to feed approximately 500 larvae per day.

\section{Preparation of Test Larvicides}

Larval bioassays experiments were conducted with Bti strain Becker Microbial Products (BMP) 144 (potency 7000 ITU [International Toxic Units]/mg) form Becker Microbial Products, Inc, (11146 NW 69 $9^{\text {th }}$ Place, Parkland, FL 33076, USA). Testing solution of Bti larvicide was prepared and serially diluted as per recommended practice. ${ }^{20}$ In brief, a stock solution was made by dissolving $200 \mathrm{mg}$ of Bti powder in $20 \mathrm{~mL}$ distilled water. The resultant $10 \mathrm{mg} / \mathrm{mL}$ stock solution was kept frozen in $2 \mathrm{~mL}$ aliquots until use. On the day of the experiment, one aliquot of the stock solution of $B t i$ was thawed and serially diluted in distilled water as recommended. Ten-fold dilution series was prepared by first transferring $2 \mathrm{~mL}$ of stock solution to $18 \mathrm{~mL}$ of chlorine-free tap water to make $1.0 \mathrm{mg} / \mathrm{mL}$ concentration, and then by subsequently repeating this procedure by transferring $2 \mathrm{~mL}$ of the latest solution to $18 \mathrm{~mL}$ of chlorine-free tap water to make $0.1,0.01$ and $0.001 \mathrm{mg} / \mathrm{mL}$ concentrations of Bti. The last three concentrations $(0.1,0.01$ and $0.001 \mathrm{mg} / \mathrm{mL}$ ) were used in the subsequent larvicide bioassays.

\section{Study design}

Larval bioassay experiments with $A$. gambiae s.s and C. quinquefasciatus were run from August to October 2019. At the beginning, two parallel larval bioassay experiments were conducted to separately test the susceptibility of A. gambiaes.s and C. quinquefasciatus larvae to Bti. They were then followed by three parallel larval bioassay experiments in which $A$. gambiae s.s and C. quinquefasciatus larvae were mixed (coexistence scenario) such that $A$. gambiae s.s contributed three quarters ( 15 out of 20 ), half ( 10 out of 20 ) and a quarter ( 5 out of 20 ) of the larvae in each testing cup (Figure 1). 


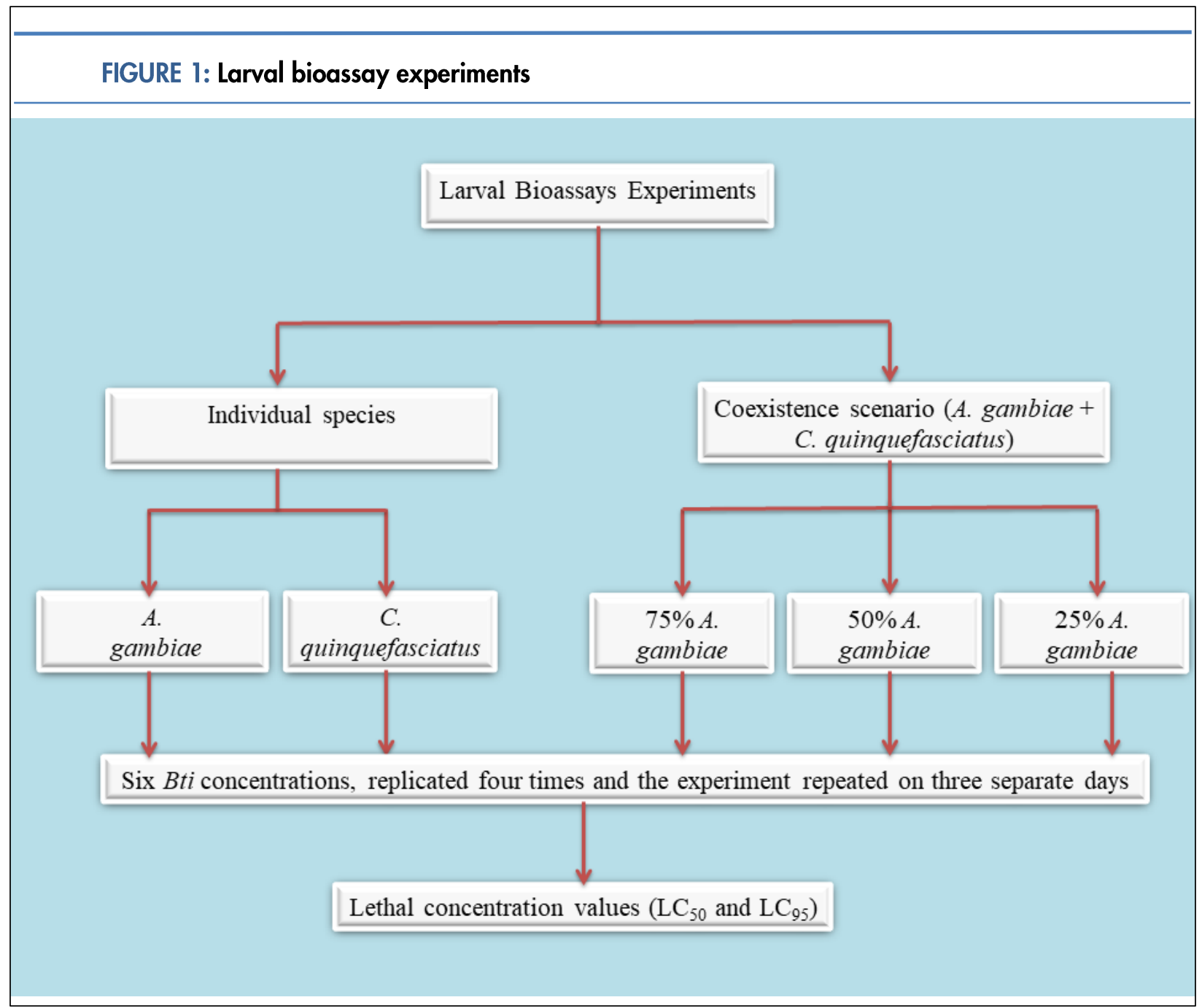

\section{Larval bioassay experiments}

At the start of each experiment, 20 third stage larvae were transferred from the larval rearing pans to the labelled disposable paper cups with $100 \mathrm{~mL}$ of chlorine-free tap water by use of disposable Pasteur pipettes. Using a pipette with disposable tips, and starting with the lowest concentration, appropriate volumes established in range finding bioassays $(0.5$ to $0.158 \mathrm{~mL}$ ) of each of the three last dilutions of Bti were then added to the experimental cups (with mosquito larvae in $100 \mathrm{~mL}$ of chlorine-free tap water). In each larval bioassay, six concentrations of Bti (including a negative control) were tested in four replicates and repeated on three different days. In negative control test cups, $0.5 \mathrm{~mL}$ of chlorine free tap water was used. The test cups were held at an average ambient temperature of $29.0^{\circ} \mathrm{C}$ and photoperiods of 12 hours light followed by 12 hours of darkness. Larval mortality was recorded at 24 hours after treatment with Bti. Only experiments with control larval mortality below $5 \%$ were included for further analysis.

\section{Data analysis}

Data on larval mortality were entered in Microsoft (MS) Excel (Microsoft Corporation, 2007) spread sheets and subsequently analysed to establish lethal concentrations (LC) of Bti that caused 50 and $95 \%$ mortality of test larvae ( $\mathrm{LC}_{50}$ and $\mathrm{LC}_{95}$ ), lethal concentration ratios (LCR) including their 95\% confidence limits by using Probit/Logit analysis software Polo Plus (2002-2003 LeOra Software, Petaluma CA, USA ${ }^{21}$. Polo Plus has been shown to be robust enough for analysis of mortality-concentration regression and its output compares fairly well with other analysis softwares. ${ }^{22}$ The variation in LCs and 
LCRs among the tested mosquitoes were compared by examining their $95 \%$ confidence limits, a common way to compare lethal concentrations or other point estimates. If the confidence limits overlap, then the LCs or LCRs do not differ significantly. ${ }^{21}$ Experiments were considered valid if control mortality did not exceed $5 \%$.

\section{RESULTS}

Overall, for the five larval bioassay experiment conducted, the lethal concentration of Bti that caused $50 \%$ and $95 \%$ mortality of the tested larvae ( $\mathrm{LC}_{50}$ and $\mathrm{LC}_{95}$ ) ranged from $0.021 \mathrm{mg} / \mathrm{L}$ to $0.065 \mathrm{mg} / \mathrm{L}$ and $0.105 \mathrm{mg} / \mathrm{L}$ to $0.423 \mathrm{mg} / \mathrm{L}$, respectively (Table 1 ). A. gambiae s.s and $C$. quinquefasciatus displayed a different level of sensitivity to Bti, with the latter species being significantly more susceptible at both $\mathrm{LC}_{50}$ and $\mathrm{LC}_{95}$ (Table 1). In the coexisting scenario, $\mathrm{LC}_{50}$ of Bti was found to be significantly lower when the proportion of $C$. quinquefasciatus exceeded $50 \%$. At $\mathrm{LC}_{95}$, there was no significant variation in susceptibility between the tested larvae at any level of coexistence, although a small trend of increased sensitivity to Bti was observed with increasing proportion of $C$. quinquefasciatus. (Table 1). These findings were confirmed by examining lethal concentration ratios of Bti calculated by comparing LC values of the tested larvae with that of $A$. gambiae s.s (Table 2). At LC $\mathrm{C}_{50}$, the lethal concentration ratio (LCR) of coexisting larvae was significantly different when the proportion of $C$. quinquefasciatus exceeded $50 \%$ and no variation in susceptibility was observed in LCR at LC 95 (Table 2). 
TABLE 1: Laboratory bioassay results of Bacillus thuringiensis var. israelensis against larvae of Anopheles gambiae s.s and Culex quinquefasciatus in different proportions of coexistence

\begin{tabular}{|c|c|c|c|c|c|c|}
\hline Mosquito species (proportion in \%) & No. tested ${ }^{\ddagger}$ & $\mathrm{LC}_{50}{ }^{\dagger}(95 \% \mathrm{CI})$ & $\mathrm{LC}_{95}{ }^{\dagger}(95 \% \mathrm{CI})$ & Slope \pm SE & $\chi^{2}(\mathrm{df})$ & Heterogeneity \\
\hline A. gambiaes.s (100) & 1440 & $0.065(0.056-0.075)$ & $0.359(0.283-0.484)$ & $2.211 \pm 0.113$ & $101.34(58)$ & 1.747 \\
\hline A. gambiaes.s $(75)+C$. quinquefasciatus (25) & 1440 & $0.059(0.053-0.067)$ & $0.423(0.345-0.540)$ & $1.925 \pm 0.097$ & $53.923(58)$ & 0.930 \\
\hline A. gambiae s.s $(50)+C$. quinquefasciatus (50) & 1440 & $0.039(0.035-0.045)$ & $0.387(0.303-0.519)$ & $1.659 \pm 0.083$ & $63.867(58)$ & 1.100 \\
\hline A. gambiae s.s $(25)+C$. quinquefasciatus (75) & 1440 & $0.038(0.033-0.042)$ & $0.300(0.241-0.391)$ & $1.821 \pm 0.090$ & $62.916(58)$ & 1.085 \\
\hline C. quinquefasciatus (100) & 1440 & $0.021(0.019-0.023)$ & $0.105(0.088-0.130)$ & $2.358 \pm 0.135$ & $52.042(58)$ & 0.897 \\
\hline
\end{tabular}

Note: ${ }^{\ddagger} 1200$ subjects and 240 controls in all tests (control mortality did not exceed $1.3 \%$ in any experiment); †mg/litre at 24 hours

Abbreviations: CI, confidence interval, SE, standard error; df, degrees of freedom, s.s, sensu stricto

TABLE 2: Lethal dose ratios for Bacillus thuringiensis var. israelensis against larvae of Anopheles gambiae s.s and Culex quinquefasciatus in different proportions of coexistence

\begin{tabular}{lcc}
\hline Mosquito species (proportion in \%) & \multicolumn{2}{c}{ Bti-Lethal Concentration Ratios ${ }^{\dagger}$ at } \\
\cline { 2 - 3 } & LC 50 (95\% CI) & LC95 $(95 \%$ CI $)$ \\
\hline A. gambiae s.s. (75) + C. quinquefasciatus (25) & $1.093(0.927-1.289)$ a & $0.848(0.626-1.147)$ a \\
A. gambiae s.s. (50) + C. quinquefasciatus (50) & $1.639(1.391-1.933)$ b & $0.928(0.672-1.281)$ a \\
A. gambiae s.s. (25) + C. quinquefasciatus (75) & $1.723(1.466-2.026)$ b & $1.195(0.881-1.621) \mathrm{a}$ \\
C. quinquefasciatus (100) & $3.101(2.674-3.597)$ c & $3.436(2.593-4.554) \mathrm{b}$
\end{tabular}

†Compared to An. gambiae sensu stricto (s.s)

Values in the columns followed by the same letter are not statistically significant (overlapping confidence interval) 


\section{DISCUSSION}

Bti has been used extensively for the control of mosquitoes and black flies. ${ }^{4,5}$ However, the activity of $B t i$ based products is affected by a multitude of factors related to the target mosquitoes, their ecology, and inherent properties of the larvicide formulations. ${ }^{5}$ of relevancy to the current study, the control efficacy of $B t i$ is known to vary with the species of the mosquito, mainly due to variation in larval feeding strategies. ${ }^{23,24}$ In larvae ecology, the coexistence of different species of mosquito larvae in aquatic habitats, particularly anopheline and culicine species is not uncommon. The current study was designed to establish whether this coexistence could affect the larvicidal activity of Bti under laboratory settings. Understanding factors that affect the activity of larvicide has both epidemiological and economic advantages in the control of mosquitoborne diseases using Bti based products.

The findings have shown that larvae of A. gambiae s.s and $C$. quinquefasciatus tested were readily susceptible to Bti at relatively low application rates, which corroborates well with the results of other studies as summarized elsewhere ${ }^{4}$. A comparison between the two species revealed that $C$. quinquefasciatus were up to three times more susceptible to Bti than A. gambiae s.s. The high sensitivity of $C$. quinquefasciatus observed in the current study has also been reported in other studies, ${ }^{12,23,24}$ and has been linked to the inherent high particulate filtration rate of this mosquito species. ${ }^{25}$ In addition to particulate filtration rate, the larvicide settling rate has been identified to impact the activity of bacterial larvicides. ${ }^{5}$ In this regard, the rapid settling rate of Bti toxins has been shown to lower larvicidal activity of surface feeding Anopheles mosquitoes. ${ }^{25,26}$ Although the settling rate of Bti was not measured in this study, the test solutions were found to settle to the bottom of storage tubes and required gentle shaking before dispensing to the testing cups. Vigorous to gentle shaking or stirring of tubes containing re-suspended bacterial larvicide preparations before application to the target larvae has been emphasized in many testing protocols. ${ }^{20}$ Although higher particulate filtration rate is known to increase the sensitivity of $C$. quinquefasciatus to Bti, it also appears likely that this species is considerably more exposed due to its bottomfeeding habit when tested with products with relatively high settling rate. The findings suggest that, if other factors that affect larval susceptibility to Bti remained constant, C. quinquefasciatus may respond better to Bti intervention than A. gambiae s.s.
In larval bioassay experiments in which larvae of $A$. gambiae and $C$. quinquefasciatus were mixed to represent various proportions of coexistence, an increased in sensitivity to Bti was recorded when compared to the $\mathrm{LC}$ values of $A$. gambiae s.s. At $\mathrm{LC}_{50}$, the increase in larval sensitivity to Bti was significantly higher when the proportion of $C$. quinquefasciatus in test cups exceeded $50 \%$. Likewise, relatively lower Bti concentrations were required to cause $95 \%$ mortality (LC95) of test larvae with increased proportion of $C$. quinquefasciatus but this did not reach statistical significance in any of the three coexistence experiments. Our findings suggest that the increase in sensitivity of coexisting larvae to Bti was possibly due to relatively high susceptibility of $C$. quinquefasciatus with an overall effect of increasing sensitivity of coexisting larvae. This assumption is supported by previous findings showing rapid onset of toxic manifestation and reduced feeding in $C$. quinquefasciatus after an initial period of feeding on Bti toxins ranging from $15-20$ minutes. ${ }^{26}$ Provided that $\mathrm{LC}_{95}$ represents the minimum effective dose by which field application rates are based, ${ }^{20}$ lack of significant variation in susceptibility of coexisting larvae at $\mathrm{LC}_{95}$ has important practical implications relevant to larviciding. In this regard, it can be safely generalized that larval control interventions using Bti can be scaled-up with little consideration to the level of coexistence between $A$. gambiae complex and $C$. quinquefasciatus.

In practical field applications, when anophelines and culicines coexist, treating the two species as a single unit in terms of susceptibility to Bti is appropriate and it has been previously documented. ${ }^{9-11}$ This approach reduces logistical challenges pertaining to resources, time and efforts that would have been required in the larvicide intervention and monitoring if coexistence had an influence on activity of Bti. Thus, this assumption allows for rapid scale-up of larviciding interventions in different ecological settings where association between anopheline and culicine species is common. Although the efficacy of Bti is affected by a multitude of factors, when these factors are considered, $B t i$ is effective and can be successfully incorporated in integrated vector management programs.

\section{CONCLUSIONS}

The findings of the current study have shown that larvae of $C$. quinquefasciatus were more susceptible to $B t i$ than those of $A$. gambiae s.s and when the two species coexisted, there was a general increase in sensitivity to Bti, which increases proportionally with C. quinquefasciatus. Although this increase in 
sensitivity of coexisting larvae to Bti is worth noting, our findings suggest that it will not impact larval control where $A$. gambiae and $C$. quinquefasciatus coexist.

\section{Authors' contributions}

YAD, EJK, WNK, GY, AKG and FWM conceived and designed the study. YAD conducted laboratory experiments and performed data analysis. YAD drafted the manuscript with contributions from EJK, WNK, GY, AKG and FWM. All authors read and approved the final version of the manuscript.

\section{Competing interests}

The authors declare that they have no competing interests.

\section{Ethical statement}

Not applicable.

\section{Availability of data and materials}

All relevant data supporting the conclusions of this article are included in the article.

Acknowledgments: The authors are grateful to the laboratory and insectary staff namely; Josephine Nyongole, Stellah Mhando, Mwanaisha Amiri, Isaya Kibwana, Justine Mnkeni, Wema Sudi, Marry Robinson, Glades Kiyaya and Grace Magogo for their technical support during mosquito rearing and larval bioassays.

\section{REFERENCE}

1. World Health Organization (WHO). World malaria report 2019. Geneva, Switzerland. 2019

2. World Health Organization (WHO). Larval source management: A supplementary measure for malaria control. An operational manual. Geneva, Switzerland. 2013

3. Derua YA, Kweka EJ, Kisinza WN, Githeko AK, Mosha FW. Bacterial larvicides used for malaria vector control in sub Saharan Africa: review of their effectiveness and operational feasibility. Parasit. Vectors 2019;12:426.

4. Walker K, Lynch M. Contribution of Anopheles larvae control to malaria suppression in tropical Africa: review of achievements and potential. Med. Vet. Entomol 2007; 21 : $2-21$.

5. Lacey LA. Bacillus thuringiensis serovariety israelensis and Bacillus sphaericus for mosquito control. Am. Mosq. Control Assoc 2007; 23, 133-163 (Suppl):S2 .

6. WHO. List of WHO Prequalified Vector Control Products. https://www.who.int/pq-vector-control/preq. (Accessed on April 14, 2020).

7. Lagadic L, Roucaute $\mathrm{M}$, Caquet $\mathrm{T}$. Bti sprays do not adversely affect non-target aquatic invertebrates in French
Atlantic coastal wetlands. J. Appl. Ecol 2014; 51: 102-13.

8. Fillinger U, Ndenga B, Githeko A, Lindsay SW. Integrated malaria vector control with microbial larvicides and insecticide-treated nets in western Kenya: a controlled trial. Bull World Health Organ. 2009;87:655-65.

9. Fillinger U, Knols BGJ, Becker N. Efficacy and efficiency of new Bacillus thuringiensis var. israelensis and Bacillus sphaericus formulations against Afrotropical anophelines in Western Kenya. Trop. Med. Int. Heal 2003;8 (1): 37-47.

10. Majambere S, Lindsay SW, GreenC, Kandeh B, Fillinger U. Microbial larvicides for malaria control in The Gambia. Malar. J 2007: 6:76.

11. Nartey R, Owusu-Dabo E, Kruppa T, Baffour-Awuah S, Annan A, Oppong S, et al. Use of Bacillus thuringiensis var. israelensis as a viable option in an integrated malaria vector control programme in the Kumasi Metropolis, Ghana. Parasit Vectors 2013; 6:116.

12. Majori G, Ali A, Sabatinelli G. Laboratory and field efficacy of Bacillus thuringiensis var. israelensis and Bacillus sphaericus against Anopheles gambiaes.l. and Culex quinquefasciatus in Ouagadougou, Burkina Faso. $J \mathrm{Am}$ Mosq Control Assoc 1987;3:20-5.

13. Kahindi SC, Midega JT, Mwangangi JM, Kibe LW, Nzovu J, Luethy P, et al. Efficacy of vectobac DT and Culinexcombi against mosquito larvae in unused swimming pools in Malindi, Kenya.J Am Mosq Control Assoc 2008; 24:53842.

14. Merritt RW, Dadd RH, Walker ED. Feeding behavior, natural food, and nutritional relationships of larval mosquitoes. Annu. Rev. Entomol 1992; 37: 349-74.

15. Aly C, Mulla MS, Xu BZ, Schnetter W. Rate of ingestion by mosquito larvae (Diptera: Culicidae) as a factor in the effectiveness of a bacterial stomach toxin. J. Med. Entomol 1988; 25(3): 191-6.

16. Fillinger U, Sonye G, Killeen GF, Knols BGJ, Becker N. The practical importance of permanent and semipermanent habitats for controlling aquatic stages of Anopheles gambiae sensu lato mosquitoes: Operational observations from a rural town in western Kenya. Trop. Med. Int. Heal 2004; 9 (12): 1274-89.

17. Matthys B, N'Goran EK, Kone M, Koudou BG, Vounatsou $P$, Cisse G,et al. Urban agricultural land use and characterization of mosquito larval habitats in a mediumsized town of Côte d'Ivoire.J. Vector Ecol 2006; 31 (2): 31933.

18. Kweka EJ, Zhou G, Beilhe LB, Dixit A, Afrane Y, Gilbreath III TM, et al. Effects of co-habitation between Anopheles gambiae s.s. and Culex quinquefasciatus aquatic stages on life history traits. Parasit Vectors 2012; 5:33.

19. Chandre, F., Darrier, F., Manga, L., Akogbeto, M., Faye, O., Mouchet, J. et al. Status of pyrethroid resistance in Anopheles gambiae sensu lato. Bull World Health Organ, 1999; 77, 230-234

20. WHO. Guidelines for laboratory and field testing of mosquito larvicides. World Health Organization Pesticides Evaluation Scheme: WHO/CDS/WHOPES/GCDPP/ 2005.13.

21. Robertson JL, Preisler HK, Russell RM. PoloPlus: Probit and Logit analysis. User's guide. 2002-2003 LeOra Software, Petaluma CA, US

22. Lei C, Sun X. Comparing lethal dose ratios using Probit regression with arbitrary slopes. BMC Pharmacol. Toxicol. 2018.19:61.

23. Aly C. Filter feeding of mosquito larvae (Diptera Culicidae) in the presence of the bacterial pathogen Bacillus thuringiensis var. israelensis. J. Appl. Entomol 1988; 105: 160-66.

24. Tyrell DJ, Davidson LI, Bulla, LA, Ramoska WA. Toxicity of parasporal crystals of Bacillus thuringiensis subsp. israelensis to mosquitoes. Appl. Environ. Microbiol 1979; 
38(4): 656-58.

25. Mullen GR, Hinkle NC. Method for determining settling rates of Bacillus thuringiensis serotype $\mathrm{H}-14$ formulations. J. Am. Mosq. Control Assoc 1988; 4(2): 132-37.

26. Standaert PJY. Persistance et l'efficacite de Bacillus thuringiensis $\mathrm{H} 14$ sur les larves de Anopheles stephensi. Zeitschrift für Angew. Entomol 1981; 91:292-300.

Peer Reviewed

Competing Interests: None declared.

Received: 10/3/2020; Accepted: 3/24/2021

Cite this article as: Derua YA Kweka EJ, Kisinza WN, Yan G, Githeko $\mathrm{AK}$, Mosha FW. The effect of the coexistence between the larvae of Anopheles gambiae and Culex quinquefasciatus on the larvicidal efficacy of Bacillus thuringiensis var. israelensis. E Afr Sci. 2021;3(1):77-85. http://doi.org/10.24248/EASci-D-20-00011

(c) Derua YA. This is an open-access article distributed under the terms of the Creative Commons Attribution License, which permits unrestricted use, distribution, and reproduction in any medium, provided the original author and source are properly cited. To view a copy of the license, visit http://creativecommons.org/licens- es/by/4.0/. When linking to this article, please use the following permanent link: http://doi.org/10.24248/EASci-D-20-00011 